\title{
Functional thermo-dynamics: A generalization of dynamic density functional theory to non-isothermal situations
}

\author{
Jesús G. Anero, ${ }^{1}$ Pep Español, ${ }^{1}$ and Pedro Tarazona ${ }^{2}$ \\ ${ }^{1}$ Dept. Física Fundamental, Universidad Nacional de Educación a Distancia, Aptdo. 60141, \\ E-28080 Madrid, Spain \\ ${ }^{2}$ Dept. Física Teórica de la Materia Condensada, Condensed Matter Physics Center IFIMAC, Universidad \\ Autónoma de Madrid, E-28049 Madrid, Spain
}

(Received 18 February 2013; accepted 5 June 2013; published online 18 July 2013)

\begin{abstract}
We present a generalization of Density Functional Theory (DFT) to non-equilibrium non-isothermal situations. By using the original approach set forth by Gibbs in his consideration of Macroscopic Thermodynamics (MT), we consider a Functional Thermo-Dynamics (FTD) description based on the density field and the energy density field. A crucial ingredient of the theory is an entropy functional, which is a concave functional. Therefore, there is a one to one connection between the density and energy fields with the conjugate thermodynamic fields. The connection between the three levels of description (MT, DFT, FTD) is clarified through a bridge theorem that relates the entropy of different levels of description and that constitutes a generalization of Mermin's theorem to arbitrary levels of description whose relevant variables are connected linearly. Although the FTD level of description does not provide any new information about averages and correlations at equilibrium, it is a crucial ingredient for the dynamics in non-equilibrium states. We obtain with the technique of projection operators the set of dynamic equations that describe the evolution of the density and energy density fields from an initial non-equilibrium state towards equilibrium. These equations generalize time dependent density functional theory to non-isothermal situations. We also present an explicit model for the entropy functional for hard spheres. (O 2013 AIP Publishing LLC. [http://dx.doi.org/10.1063/1.4811655]
\end{abstract}

\section{INTRODUCTION}

In his foundation of statistical mechanics, ${ }^{1}$ Gibbs introduced the notion of ensembles in phase space as a tool for describing the macroscopic properties of matter in terms of its molecular structure. He made thorough use of the concept of entropy in order to construct the equilibrium ensembles. Much later, Jaynes ${ }^{2}$ advocated under the name of the Principle of Maximum Entropy (PME) the information theoretical point of view that regards the ensembles as those probability distributions that are the least biased according to the available macroscopic information. Although the PME allows one to construct equilibrium ensembles, it has an even larger scope because it allows to construct dynamic theories that describe not only the equilibrium state but also the tendency towards equilibrium starting from a non-equilibrium state. This is achieved by separating the time dependent ensemble that obeys the Liouville equation in two contributions, a relevant ensemble (or local equilibrium ensemble) that represents the least biased information that is compatible with the time dependent macroscopic information, and a remaining contribution. ${ }^{3}$ By solving for the remaining contribution, a closed equation for the relevant ensemble is obtained and, as a consequence, a closed exact dynamic equation for the macroscopic information. This process may be implemented with the help of projection operators and the resulting theory is usually referred as the Mori-Zwanzig theory, ${ }^{4,5}$ although the number of important contributions by different authors is very large. ${ }^{3,6-11}$
One important concept in statistical mechanics is that of level of description of a given system. A level of description is characterized by a set of phase functions named relevant variables (or macrostates, or macroscopic variables, or collective variables, or coarse-grained (CG) variables, or reaction coordinates, etc.). A given system may be described at different levels of description and each level has characteristic timescales. At the coarsest level, one has Macroscopic Thermodynamics (MT) characterized by the dynamical invariants of the system. While the theory of MT summarizes an enormous number of experimental observations, it is limited by the fact that it deals with big homogeneous portions of material. ${ }^{12}$ In order to study equilibrium states statistically inhomogeneous at molecular scales, the powerful density functional theory (DFT) was formulated in the second half of last century. ${ }^{13}$ DFT allows one to describe the structures of fluids and solids and their phase changes with a very high accuracy. In this way, a wealth of phenomena ranging from wetting of liquids on surface ${ }^{14}$ to phase transitions in solids ${ }^{15-17}$ can be addressed. DFT is based on a theorem first enunciated by Mermin. ${ }^{18}$ Mermin's theorem ${ }^{18}$ has two parts, in fact, that can be understood as independent theorems. In the first part, it is shown that the connection between the one-particle distribution function, i.e., the average density field, and any external potential is bijective. For every external potential there is a unique density profile and vice-versa. The proof requires the construction of a functional of the density field named the density functional. The second part of the theorem shows that 
one can obtain the macroscopic free energy of the system by evaluating the density functional at the equilibrium value of the density field. This equilibrium density is the one that minimizes the density functional. In the present paper, we show that Mermin theorem ${ }^{18}$ can be generalized substantially and that it emerges basically from the principle of maximum entropy. We show that every level of description has its own entropy function and describe how the different entropies of each level of description are related to each other.

We focus in the present paper on a level of description named Functional Thermo-Dynamics (FTD), which is characterized by the density field and the energy density field as relevant variables. One of the basic objects of the theory of the FTD level of description is the entropy functional, which is a functional of the density and energy density fields and that contains, in very much the same way as the density functional of DFT, all the information about the molecular structure of the fluid/solid system. When applied to a homogeneous equilibrium state, DFT recovers the thermodynamic magnitudes of MT, but it goes beyond it, since from DFT we may extract the spatial correlations between the particles in a homogeneous system. Similarly, when applied to a (homogeneous or inhomogeneous) equilibrium state, the FTD reproduces all the results of DFT, but it opens the access to the time evolution of the relevant variables and allows to describe dynamical non-isothermal situations.

The construction of dynamic equations based on DFT has been a subject of high interest in recent years. This dynamic density functional theory (DDFT) has been constructed under different approaches an approximations. Usually, colloidal suspensions governed by a Smoluchovski overdamped dynamics have been considered in the framework of DDFT. ${ }^{19-21}$ Also, kinetic theory has been used as starting point for the derivation of DDFT. ${ }^{22-27}$ Recently, Hughes and Burghardt ${ }^{28}$ have developed a hydrodynamic theory that includes correlations starting from a kinetic equation in which the collision operator is modelled phenomenologically with a Fokker-Planck operator. The use of the maximum entropy principle in order to close hierarchies of dynamic equations based on a kinetic description has been considered in a number of works ${ }^{29,30}$ We have contributed to the field by deriving DDFT from projection operators ${ }^{31}$ starting from the underlying Hamiltonian dynamics. We pursue further in the present paper this line by using the general theory of projection operators ${ }^{32}$ in order to construct the dynamic theory that describes the evolution in time of the density and energy density fields for simple fluids at molecular scales.

There is very recent and exciting work addressing the extension of DDFT towards non-isothermal situations. In particular, Schmidt has presented a formulation based on an internal energy functional that depends on the number and entropy density fields, both defined in microscopic terms. ${ }^{33}$ From this general and rigorous formulation, he proposes phenomenological transport equations for these variables within the framework of linear irreversible thermodynamics. It is of high interest to provide a microscopic basis for the phenomenological approach and, in particular, provide for molecular expressions for the transport coefficients appearing in the transport equations. This is the approach that we take in the present work where it turns out that an entropy functional, instead of an internal energy functional, is the natural functional within the technique of projection operators from which the transport equations are derived in a straightforward manner from the microscopic dynamics. Very recently Wittkowski, Löwen, and Brand have presented a similar theory based on projection operators, which addresses the non-isothermal dynamics of mixtures of colloidal particles. ${ }^{34}$

\section{GENERAL FRAMEWORK}

\section{A. Macrocanonical phase space}

Let us consider an isolated system made of $N$ classical particles moving according to Hamilton's equations with a time-independent Hamiltonian. The microstate of the system is $z_{N}$ corresponding to the positions and momenta of all the particles. The collection of all microstates $z_{N}$ is the phase space $\Gamma_{N}$. As it is well-known, the fact that we cannot specify the initial conditions of a system of many degrees of freedom with absolute precision requires the use of a probability density $\rho_{N}\left(z_{N}\right)$ in phase space. Note that in many instances we do not even know with exact precision how many particles constitute our system. In that case, we need to use the union of all phase spaces $\Gamma_{N}$ with different number of particles. The microstate is specified by saying in what $\Gamma_{N}$ the system is and what particular $z_{N}$ of that $\Gamma_{N}$ the system has. We may call the collection of phase spaces $\Gamma=\left\{\Gamma_{N}, N\right.$ $=1, \ldots, \infty\}$, the macrocanonical phase space, with the proviso that we are not dealing with any exchange of particles with a reservoir but rather with our ignorance about the number of particles that constitute our system. The probability density is normalized in such a way that $P(N)=\int d z_{N} \rho_{N}\left(z_{N}\right)$ is the probability of finding the system in the phase space of $N$ particles. The full set of probability densities $\left\{\rho_{N}\left(z_{N}\right)\right.$, $N=1, \ldots, \infty\}$ gives the statistical description of the system at the microscopic level. In each phase space, $\Gamma_{N}$ the probability density $\rho_{N}\left(z_{N}, t\right)$ evolves according to the corresponding Liouville equation $\partial_{t} \rho_{N}\left(z_{N}, t\right)=i L_{N} \rho_{N}\left(z_{N}, t\right)$ with its own $N$-particle Liouville operator. This implies that $\partial_{t} P(N)$ $=\partial_{t} \int d z_{N} \rho_{N}\left(z_{N}, t\right)=0$. There are no "jumps" in the dynamics from one phase space to another and the probability of having a given number of particles is time independent. This is another way to see that the system does not exchange particles with any reservoir. The averages of phase functions of $N$ particles are defined as

$$
\langle F\rangle_{t}=\sum_{N=1}^{\infty} \int d z_{N} F_{N}\left(z_{N}\right) \rho_{N}\left(z_{N}, t\right) .
$$

Here, $F_{N}\left(z_{N}\right)$ is a phase function defined in the phase space of $N$ particles. One example of these phase functions is the Hamiltonian $H_{N}\left(z_{N}\right)$. The above average has obviously a "macrocanonical look." Usually, we will denote with a trace the sum over microstates, that is,

$$
\operatorname{Tr}[\cdots]=\sum_{N=1}^{\infty} \int d z_{N} \cdots
$$


The system may be described at different levels of description depending on the amount of information available macroscopically or mesoscopically. A level of description is fully determined by a set of functions in the phase space of the system. We denote the macrostate with a set of phase functions $A(z)=\left\{A_{N}\left(z_{N}\right), N=1, \ldots, \infty\right\}$. The variables $A(z)$ that characterize a given level of description will be referred to as relevant variables or coarse-grained variables. The coarsegrained variables $A(z)$ may be discrete variables or fields, and they may have vector or tensorial character.

\section{B. The relevant ensemble and the entropy of a level of description}

We will construct in this paper the entropy associated to several levels of description by using the maximum entropy principle first used by Gibbs. ${ }^{1}$ This well-known method introduces the entropy functional of the ensemble density as

$$
\begin{aligned}
\mathcal{S}[\rho] & =-\operatorname{Tr}\left[\rho(z) \ln \left(\frac{\rho(z)}{\rho^{0}}\right)\right] \\
& =-\sum_{N=1}^{\infty} \int d z_{N} \rho_{N}(z) \ln \frac{\rho_{N}(z)}{\rho_{N}^{0}},
\end{aligned}
$$

where the quantum mechanical factor $\rho_{N}^{0}=1 /\left(h^{3 N} N\right.$ !) gives the correct dimensionless form to the argument of the logarithm an ensures the correct Boltzmann counting and proper account of the indistinguishability of particles. Usually the Boltzmann constant $k_{B}$ appears in front of (3). By not including this factor, we are measuring the entropy functional in units of $k_{B}$.

The basic idea of the maximum entropy principle ${ }^{2}$ is that in order to find the least biased probability density $\rho(z)$ in phase space (i.e., the least biased ensemble) when only partial information is known about $\rho(z)$, one should maximize the entropy functional (3) subject to the constraints of this partial information. The entropy functional represents, from an information theory point of view, the "amount of uncertainty" of a distribution function ${ }^{2}$ and by maximizing it subject to whatever is known, we are taking the distribution which is "maximally noncommittal with regard to missing information, and that it agrees with what is known, but expresses maximum uncertainty with respect to all other matters" in Jaynes words. ${ }^{2}$ The ensemble that maximizes the entropy (3) subject to the partial information that we have about the system is named the relevant ensemble $\bar{\rho}(z)$.

We will consider in this paper that the partial information known about the ensemble $\rho(z)$ is given by the average $a$ of the relevant variables $A(z)$, that is,

$$
\operatorname{Tr}[\rho(z) A(z)]=a .
$$

Many $\rho(z)$ are compatible with this restriction. The least biased is the one that maximizes (3). Standard maximization of (3) with the constraint (4) and the normalization of $\rho(z)$ leads to the relevant ensemble

$$
\bar{\rho}(z)=\frac{\rho^{0}}{Z[\lambda]} \exp \{-\lambda A(z)\} .
$$

This is a condensed notation for the collection of probability densities,

$$
\bar{\rho}_{N}\left(z_{N}\right)=\frac{\rho_{N}^{0}}{Z[\lambda]} \exp \left\{-\lambda A_{N}\left(z_{N}\right)\right\} \quad N=1, \cdots \infty .
$$

Also, $\lambda A_{N}\left(z_{N}\right)$ should be understood as the scalar product in a general sense of the conjugate variables $\lambda$ and the CG variables $A_{N}\left(z_{N}\right)$. For example, in the case of field CG variables, there is an integral over space of the two fields $\lambda$ and $A_{N}\left(z_{N}\right)$. In the case of vector or tensor variables, full contraction over the indices is implied so as to produce a scalar. The dimensionless partition function is defined as

$$
Z[\lambda] \equiv \operatorname{Tr}\left[\rho^{0} \exp \{-\lambda A(z)\}\right] .
$$

We assume that the phase functions $A(z)$ are such that the integral in the partition function actually exists. The Lagrange multiplier $\lambda$ is obtained by requiring that the generalized canonical ensemble (5) fulfills the constraint (4). This requirement can be written as

$$
\frac{\partial \Omega[\lambda]}{\partial \lambda}=a,
$$

where we have introduced the dimensionless thermodynamic potential of the present level of description as

$$
\Omega[\lambda]=-\ln Z[\lambda] .
$$

The implicit equation (8) allows one to obtain $\lambda(a)$. In fact, there is a one to one correspondence between $\lambda$ and $a$, as can be shown by taking the derivative of Eq. (8) with respect to $\lambda$ :

$$
\begin{aligned}
\frac{\partial^{2} \Omega[\lambda]}{\partial \lambda \partial \lambda} & =-\langle\delta A \delta A\rangle^{\lambda} \\
& =-\sum_{N=1}^{\infty} \int d z_{N} \delta A_{N}\left(z_{N}\right) \delta A_{N}\left(z_{N}\right) \bar{\rho}_{N}\left(z_{N}\right),
\end{aligned}
$$

where $\delta A=A(z)-a$ and the average $\langle\cdots\rangle^{\lambda}$ is performed with $\bar{\rho}(z)$. This shows that $\Omega[\lambda]$ is a concave functional of $\lambda$ because the Hessian matrix of second derivatives, being the negative of a covariance, is negative definite. ${ }^{35,36}$ Equation (10) also means that the Jacobian of the change of variables from $\lambda$ to $a$ is

$$
\frac{\partial a}{\partial \lambda}=-\langle\delta A \delta A\rangle^{\lambda} .
$$

The covariance $\langle\delta A \delta A\rangle^{\lambda}$ is a definite positive matrix, and, therefore, the Jacobian of the change of variables from $\lambda$ to $a$ can be inverted to provide $\lambda[a]$. Therefore, there is a one to one connection between the pair of conjugate variables $\lambda$ and $a$. This argument is valid for any pair of conjugate variables. Mermin's theorem ${ }^{18}$ is a particular case of this general case for the DFT level of description ensuring that there is a one to one connection between the density field and the external potential (or chemical potential).

The fact that second derivatives of the thermodynamic potential are a covariance matrix ensures that they form a positive semi-definite matrix. For arbitrary relevant variables, it could happen that $A v=0$ for some vector $v$. If this is the case, then the covariance matrix has a zero eigenvalue and is not, strictly speaking, invertible. Note that, in this case, the partition function satisfies $Z[\lambda]=Z[\lambda+\alpha v]$ for any scalar $\alpha$. 
Therefore, the thermodynamic potential $\Omega[\lambda]$ is translationally invariant in the direction of $v$. In the space of $\lambda$, the thermodynamic potential has a "canyon" shape in the direction of $v$ and not a "hump" with a global maximum. Nevertheless, one still has a one to one connection between the averages $a$, that live in the plane $a v=0$, and the parameters $\lambda$ that live in the plane $\lambda v=0$. In this subspace, one has strict concavity of the thermodynamic potential.

Once the relevant ensemble has been obtained, the entropy function (or functional) at a given level of description is defined by the result of evaluating the Gibbs entropy functional (3) at the relevant ensemble (5), with the result

$$
S[a] \equiv \mathcal{S}[\bar{\rho}]=-\Omega[\lambda[a]]+\lambda[a] a .
$$

We observe that the entropy and the dimensionless thermodynamic potential are Legendre transform of each other (up to signs). By taking the derivative of (12) with respect to $a$ and using Eq. (8), we have the expression conjugate to Eq. (8),

$$
\frac{\partial S[a]}{\partial a}=\lambda[a] .
$$

It should be clear that the framework presented has nothing to do with equilibrium states. In particular, the relevant ensemble $\bar{\rho}$ is not an equilibrium ensemble in general and, therefore, $a$ are not the equilibrium averages of the CG variables $A(z)$. Only if the relevant variables are the dynamical invariants of the microscopic dynamics then the relevant ensemble, that depends on the microstate through these dynamical invariants, is a stationary solution of the Liouville equation and, therefore, an equilibrium ensemble.

\section{The bridge theorem}

In this section, we show how the entropies (12) of two levels of description are related. We consider two levels of description characterized by the set of phase functions $Y(z)$, $X(z)$. We assume that $X(z)=\mathcal{X}(Y(z))$ and, therefore, the $x$-level is less detailed than the $y$-level of description (which, in turn, is less detailed than the microscopic level described by $z$ ). We may refer to $y$ as fine grained (FG) variables and $x$ as CG variables. The basic question that we want to answer in this subsection is what is the connection between the functional form of the entropy $S_{x}(x)$ and the entropy $S_{y}(y)$ of each level of description? Although we may consider a general functional $\mathcal{X}(y)$ form connecting the relevant variables of different levels of description, we restrict ourselves to a linear relationship of the form

$$
X(z)=A Y(z)
$$

where $A$ is a rectangular constant matrix. It turns out that this connection between levels of descriptions is, in fact, quite common and, in particular, the levels of MT, DFT, and FTD have relevant variables connected in a linear way.

The maximization of the entropy functional (3) is subject to give prescribed averages $y=\operatorname{Tr}[\rho Y]$ or $x=\operatorname{Tr}[\rho X]$, which give the two relevant ensembles of each level of description:

$$
\begin{aligned}
& \bar{\rho}_{y}(z)=\rho^{0} \frac{\exp \left\{-\lambda_{y} Y(z)\right\}}{Z_{y}\left(\lambda_{y}\right)}, \\
& \bar{\rho}_{x}(z)=\rho^{0} \frac{\exp \left\{-\lambda_{x} X(z)\right\}}{Z_{x}\left(\lambda_{x}\right)},
\end{aligned}
$$

where $\lambda_{y}$ and $\lambda_{x}$ are the Lagrange multiplier enforcing the constraints,

$$
\begin{aligned}
& y=\operatorname{Tr}\left[\bar{\rho}_{y} Y\right], \\
& x=\operatorname{Tr}\left[\bar{\rho}_{x} X\right],
\end{aligned}
$$

respectively. In addition, we will enforce that the averages are related according to $x=A y$. This implies, for example, that the average of the CG variable $X(z)$ with respect to the relevant ensemble of the FG $y$-level is precisely $x$, that is,

$$
\operatorname{Tr}\left[\bar{\rho}_{y} X\right]=x \text {. }
$$

This is a condition on the possible values that $y$ may take, given $x$.

The entropies of each level of description are given by (12), which now take the form:

$$
\begin{aligned}
& S_{y}(y)=\ln Z_{y}(y)+\lambda_{y}(y) y, \\
& S_{x}(x)=\ln Z_{x}(x)+\lambda_{x}(x) x .
\end{aligned}
$$

Consider now the maximum $y^{*}$ of $S_{y}(y)$ subject to the restriction (17). The state $y^{*}$ is obviously a function of $x$ appearing in the restriction (17). It is easily shown that by evaluating $S_{y}(y)$ at $y^{*}$, we obtain precisely $S_{x}(x)$, that is,

$$
S_{y}\left(y^{*}(x)\right)=S_{x}(x) .
$$

In order to prove this, let us maximize without constraints the function,

$$
\begin{aligned}
S_{y}(y)-\mu \operatorname{Tr}\left[\bar{\rho}_{y} X\right] & =S_{y}(y)-\mu \operatorname{Tr}\left[\bar{\rho}_{y} A Y\right] \\
& =S_{y}(y)-\mu A y,
\end{aligned}
$$

where $\mu$ is the set of Lagrange multipliers enforcing (17). The derivative of this function equated to zero is

$$
\lambda_{y}\left(y^{*}\right)-\mu A=0 .
$$

This is an implicit equation for $y^{*}$ that will depend parametrically on $\mu$. The actual value of $\mu$ is obtained by requiring the fulfilment of the constraint (17) that now becomes

$$
\operatorname{Tr}\left[\bar{\rho}_{y^{*}} A Y\right]=x \rightarrow A y^{*}=x .
$$

In this way, for every particular $x$, we have a given $\mu(x)$. Finally, the entropy of the $y$-level evaluated at this particular maximum becomes

$$
\begin{aligned}
S_{y}\left(y^{*}\right) & =\ln Z_{y}\left(\lambda_{y}\left(y^{*}\right)\right)+\lambda_{y}\left(y^{*}\right) y^{*} \\
& =\ln \operatorname{Tr}\left[\exp \left\{-\lambda_{y}\left(y^{*}\right) Y\right\}\right]+\lambda_{y}\left(y^{*}\right) y^{*} \\
& =\ln \operatorname{Tr}[\exp \{-\mu(x) A Y\}]+\mu(x) A y^{*} \\
& =\ln \operatorname{Tr}[\exp \{-\mu(x) X\}]+\mu(x) x \\
& =\phi(x) .
\end{aligned}
$$


We have to still prove that $\phi(x)=S_{x}(x)$. Consider again (22) now with the relevant ensemble $\bar{\rho}_{y^{*}}(15)$ corresponding to $y^{*}$, that is,

$$
\frac{\operatorname{Tr}\left[\rho_{0} \exp \left\{-\lambda_{y}\left(y^{*}\right) Y\right\} X\right]}{\operatorname{Tr}\left[\rho_{0} \exp \left\{-\lambda_{y}\left(y^{*}\right) Y\right\}\right]}=x .
$$

Use Eq. (21) in this last equation to obtain

$$
\frac{\operatorname{Tr}\left[\rho_{0} \exp \{-\mu(x) X\} X\right]}{\operatorname{Tr}\left[\rho_{0} \exp \{-\mu(x) X\}\right]}=x .
$$

This is to be compared with the second equation in (16) that takes the form:

$$
\frac{\operatorname{Tr}\left[\rho_{0} \exp \left\{-\lambda_{x}(x) X\right\} X\right]}{\operatorname{Tr}\left[\rho_{0} \exp \left\{-\lambda_{x}(x) X\right\}\right]}=x .
$$

Because the connection between the averages $x$ and the conjugate variables $\lambda_{x}(x)$ is unique, it follows that $\mu(x)=\lambda_{x}(x)$ and, therefore, $\phi(x)=S_{x}(x)$, that is,

$$
S_{y}\left(y^{*}\right)=S_{x}(x) .
$$

The bridge theorem can be expressed in very compact form as follows:

$$
\max _{\operatorname{Tr}[\rho A y]=x} \mathcal{S}[\rho]=\max _{x=A y}\left[\max _{\operatorname{Tr}[\rho Y]=y} \mathcal{S}[\rho]\right] .
$$

In words, the left-hand side finds the maximum of the Gibbs entropy functional $\mathcal{S}[\rho]$ for those ensembles in the subset satisfying $\operatorname{Tr}[\rho Y]=y$ and then the maximum in the smaller, but fully contained subset of ensembles fulfilling $x=A y$, while the right-hand side finds the maximum in the set $\operatorname{Tr}[\rho A y]$ $=x$ directly. Dwandaru and Schmidt ${ }^{37}$ have recently considered this double maximization process when relating the DFT and MT levels of description. In the entropy language, their Eqs. (43) and (44) in Ref. 37 become

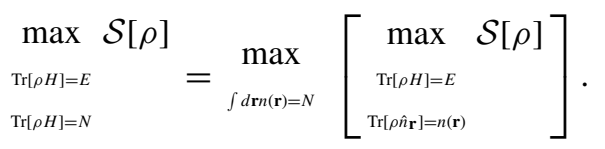

The left-hand side is just the usual macroscopic thermodynamic entropy, while the term inside brackets of the righthand side is the entropy functional at the DFT level of description. Equation (29) is just a particular realization of the general bridge theorem (28).

In conclusion, if we know the entropy of a FG level of description, we automatically know the entropy of any CG level of description whose $\mathrm{CG}$ variables are linear combinations of the FG variables. The recipe is simple: maximize the entropy of the FG level subject to the constraint that the average of the FG variables gives the CG variables, and substitute back this maximum value into the entropy of the FG level. The result is the entropy at the CG level.

The bridge theorem (23) is a generalization of the second part of Mermin's theorem ${ }^{18}$ that allows one to connect the entropies of different levels of descriptions provided that the relevant variables of each level are connected in a linear functional form. When the level of description is DFT the above theorem is just the original Mermin theorem. ${ }^{18}$ The bridge theorem (23) can be of great importance as a guiding tool for the construction of the entropy functional of detailed levels of description if we know the corresponding entropy at CG levels of description (like macroscopic thermodynamics or density functional theory). In a similar way, it allows to compute the entropy of MT from molecular models that we may construct for the entropy at the DFT or FTD levels of description.

\section{The time-dependent projection operator technique}

For completeness, we summarize in this section the timedependent projection operator technique as presented in the classical textbook by Grabert. ${ }^{32}$ This is the method that we will use to obtain the dynamic equations for the relevant variables. While the method is described in Ref. 32 for a fixed number $N$ of particles, it is valid also for the macrocanonical phase space by just understanding the trace symbol according to Eq. (2). The method provides formally exact evolution equation for any arbitrary dynamic variable. Physics enters in the theory by the very selection of the relevant variables, which are in general assumed to be slowly varying in time. The projection operator method can be understood, at its most fundamental level, as a way to approximate the actual time dependent ensemble $\rho_{t}$, which is the solution of the Liouville equation with an approximate ensemble $\bar{\rho}_{t}$ of the relevant type (5). ${ }^{3}$ The aim is to derive equations of motion for the time dependent average $a_{i}(t)$ of a set of relevant variables $A_{i}(z)$. The time dependent average is

$$
a_{i}(t)=\operatorname{Tr}\left[\rho_{t}(z) A_{i}(z)\right],
$$

where $z$ is the microscopic state of the system and $\rho_{t}(z)$ is the non-equilibrium solution of the Liouville equation. As it is shown in Ref. 32, the Liouville equation can be re-written in the following exact form:

$$
\frac{\partial}{\partial t} a_{i}(t)=v_{i}(t)+\int_{0}^{t} d t^{\prime} \sum_{j} K_{i j}\left(t, t^{\prime}\right) \lambda_{j}(t) .
$$

This exact equation is actually a closed equation for $a_{i}(t)$. The reversible term is given by

$$
v_{i}(t)=\operatorname{Tr}\left[\bar{\rho}_{t} i L A_{i}\right],
$$

where $i L$ is the Liouville operator and the relevant ensemble $\bar{\rho}_{t}$ is of the form (5), with a time dependent conjugate variable $\lambda(t)$. The conjugate variables $\lambda$ are selected in such a way that the averages of $A(z)$ performed with the real and with the relevant ensemble coincide. The conjugate variables are, through Eq. (13), the derivatives of the entropy function of the given level of description. Note that if only the reversible term $v_{i}(t)$ would be present in Eq. (31), we would be approximating the actual ensemble that it is a solution of the Liouville equation with a relevant ensemble of the form (5) where the conjugate field $\lambda(t)$ is now a function of time. The error in this approximation is, in fact, the memory term which describes irreversible behaviour. The irreversible term in Eq. (31) involves the memory kernel $K_{i j}\left(t, t^{\prime}\right)$ whose explicit form can be found in Ref. 32. Equation (31) is a closed exact equation for $a_{i}(t)$ but its integro-differential character makes it difficult to treat in general. Nevertheless, the exact transport equation (31) can be approximated by a memory-less equation whenever a clear separation of time scales exists between the evolution of the 
averages and the decay of the memory kernel. In this case, we assume that, within the time in which the kernel decays, the relevant variables have not changed appreciably. Under this assumption, one obtains the Markovian equation, ${ }^{32}$

$$
\dot{a}_{i}(t)=v_{i}(t)+\sum_{j} D_{i j}(t) \frac{\partial S}{\partial a_{j}}(t)
$$

where we have used (13) and where the dissipative matrix is given by the Green-Kubo formula,

$$
D_{i j}(t)=\int_{0}^{\infty} d t^{\prime} \operatorname{Tr}\left[\bar{\rho}_{t}\left(\mathcal{Q}_{t} i L A_{j}\right) G_{t t^{\prime}}\left(\mathcal{Q}_{t} i L A_{i}\right)\right]
$$

where the so-called projected current is given by

$$
\begin{aligned}
\mathcal{Q}_{t^{\prime}} i L A_{k}(z)= & i L A_{k}(z)-\operatorname{Tr}\left[\bar{\rho}_{t^{\prime}} i L A_{k}\right] \\
& -\left[A_{i}(z)-a_{i}\left(t^{\prime}\right)\right] \frac{\partial v_{k}\left(t^{\prime}\right)}{\partial a_{i}\left(t^{\prime}\right)} .
\end{aligned}
$$

$G_{t^{\prime} t}$ is given by a time ordered projected propagator. ${ }^{32}$ Under the assumption that the relevant variables are slow, one can take an approximation in which terms of order $i L A^{3}$ are neglected. The result of this procedure is that the projected dynamics $G_{t t^{\prime}}$ is approximated by the actual dynamics exp $\{i L t\}$ (see Ref. 38 for an alternative approach). Note that the dissipative matrix in Eq. (34) depends in general on the relevant variables through the relevant ensemble. The dissipative matrix $D_{i j}$ is a correlation matrix and, due to the Wiener- Khintchine theorem, it is positive definite. ${ }^{32}$ This leads to a very general feature of the dynamic equation (33) obtained with the projection operator method: They automatically ensure that the entropy (12) of the given level of description is always a non-decreasing function of time. Indeed, by taking the time derivative of the entropy $S(a)$, using the chain rule and the dynamic equation (33), and noting that the drift term $v_{i}(a)$ gives a vanishing contribution, ${ }^{32}$ we obtain

$$
\dot{S}(a)=\sum_{i j} \lambda_{i} D_{i j} \lambda_{j} \geq 0,
$$

which is a reflection of the second law of thermodynamics for the given level of description.

\section{FUNCTIONAL THERMODYNAMICS}

In this section, we consider the level of description of FTD, which is specified by the averages of the density field and the energy density field. The microscopic expressions of these variables are

$$
\begin{aligned}
& \hat{n}_{\mathbf{r}}(z)=\sum_{i=1}^{N} \delta\left(\mathbf{r}-\mathbf{r}_{i}\right), \\
& \hat{e}_{\mathbf{r}}(z)=\sum_{i=1}^{N} e_{i} \delta\left(\mathbf{r}-\mathbf{r}_{i}\right) .
\end{aligned}
$$

Here, $e_{i}$ is the energy of particle $i$, which is defined as

$$
e_{i}=\frac{\mathbf{p}_{i}^{2}}{2 m_{i}}+\frac{1}{2} \sum_{j \neq i} \phi\left(r_{i j}\right)+V^{\mathrm{ext}}\left(\mathbf{r}_{i}\right)
$$

where $\phi(r)$ is the (pair-wise) interparticle potential and $V^{\text {ext }}(\mathbf{r})$ is any external potential acting on the particles. The averages of these phase functions with respect to the relevant distribution in the macrocanonical phase space are denoted by

$$
\begin{aligned}
& n(\mathbf{r})=\sum_{N=0}^{\infty} \int d z_{N} \bar{\rho}_{N}\left(z_{N}\right) \hat{n}_{\mathbf{r}}\left(z_{N}\right), \\
& e(\mathbf{r})=\sum_{N=0}^{\infty} \int d z_{N} \bar{\rho}_{N}\left(z_{N}\right) \hat{e}_{\mathbf{r}}\left(z_{N}\right) .
\end{aligned}
$$

The relevant ensemble (5) has the form

$$
\bar{\rho}_{N}(z)=\frac{\rho^{0}}{Z_{\mathrm{FTD}}[\lambda, \beta]} \exp \left\{-\int d \mathbf{r}\left(\lambda(\mathbf{r}) \hat{n}_{\mathbf{r}}(z)+\beta(\mathbf{r}) \hat{e}_{\mathbf{r}}(z)\right)\right\} .
$$

Here, $\lambda(\mathbf{r})$ and $\beta(\mathbf{r})$ are the Lagrange multipliers associated to each constraint in (39). The normalization factor is the dimensionless partition function defined as

$$
Z_{\mathrm{FTD}}[\lambda, \beta]=\sum_{N=0}^{\infty} \int d z_{N} \rho^{0} \exp \left\{-\sum_{i=1}^{N}\left(\lambda\left(\mathbf{r}_{i}\right)+\beta\left(\mathbf{r}_{i}\right) e_{i}\right)\right\} .
$$

This partition function is a functional of both fields $\lambda, \beta$, which are the conjugate fields of the number density and energy density, respectively. By inserting (40) into (39), we may find the connection between the Lagrange multipliers $\lambda(\mathbf{r})$, $\beta(\mathbf{r})$ and the averages $n(\mathbf{r}), e(\mathbf{r})$. This connection can be expressed as

$$
\begin{aligned}
& \frac{\delta \Omega_{\mathrm{FTD}}[\lambda, \beta]}{\delta \lambda(\mathbf{r})}=n(\mathbf{r}), \\
& \frac{\delta \Omega_{\mathrm{FTD}}[\lambda, \beta]}{\delta \beta(\mathbf{r})}=e(\mathbf{r}),
\end{aligned}
$$

where the dimensionless thermodynamic potential of the functional thermodynamics level of description is given by

$$
\Omega_{\mathrm{FTD}}[\lambda, \beta] \equiv-\ln Z_{\mathrm{FTD}}[\lambda, \beta]
$$

By taking functional derivatives of Eqs. (42) with respect to $\lambda(\mathbf{r}), \beta(\mathbf{r})$, we obtain

$$
\begin{aligned}
& \frac{\delta^{2} \Omega_{\mathrm{FTD}}[\lambda, \beta]}{\delta \lambda(\mathbf{r}) \delta \lambda\left(\mathbf{r}^{\prime}\right)}=\left\langle\delta n_{\left.\mathbf{r} \delta n_{\mathbf{r}^{\prime}}\right\rangle^{[\lambda, \beta]},}\right. \\
& \frac{\delta^{2} \Omega_{\mathrm{FTD}}[\lambda, \beta]}{\delta \lambda(\mathbf{r}) \delta \beta\left(\mathbf{r}^{\prime}\right)}=\left\langle\delta n_{\mathbf{r}^{\prime}} \delta e_{\mathbf{r}^{\prime}}\right\rangle^{[\lambda, \beta]}, \\
& \frac{\delta^{2} \Omega_{\mathrm{FTD}}[\lambda, \beta]}{\delta \beta(\mathbf{r}) \delta \beta\left(\mathbf{r}^{\prime}\right)}=\left\langle\delta e_{\mathbf{r}} \delta e_{\mathbf{r}^{\prime}}\right\rangle^{[\lambda, \beta]} .
\end{aligned}
$$

The average $\langle\cdots\rangle^{[\lambda, \beta]}$ is performed with the relevant ensemble (40). Note that the Hessian matrix of second derivatives is given in terms of covariances performed with the relevant ensemble. The covariance matrix is a strictly positive definite matrix in the following sense. Take a "vector" of the form 
$\left(v_{\mathbf{r}}, u_{\mathbf{r}}\right)$ and construct the quadratic form

$$
\begin{gathered}
\int d \mathbf{r} \int d \mathbf{r}^{\prime}\left(v_{\mathbf{r}}, u_{\mathbf{r}}\right)\left(\begin{array}{cc}
\left\langle\delta n_{\mathbf{r}} \delta n_{\mathbf{r}^{\prime}}\right\rangle^{[\lambda, \beta]} & \left\langle\delta n_{\mathbf{r}} \delta e_{\mathbf{r}^{\prime}}\right\rangle^{[\lambda, \beta]} \\
\left\langle\delta e_{\mathbf{r}} \delta n_{\mathbf{r}^{\prime}}\right\rangle^{[\lambda, \beta]} & \left\langle\delta e_{\mathbf{r}} \delta e_{\mathbf{r}^{\prime}}\right\rangle^{[\lambda, \beta]}
\end{array}\right)\left(\begin{array}{c}
v_{\mathbf{r}^{\prime}} \\
u_{\mathbf{r}^{\prime}}
\end{array}\right) \\
=\left\langle\left(\int d \mathbf{r}\left(v_{\mathbf{r}} \delta n_{\mathbf{r}}+u_{\mathbf{r}} \delta e_{\mathbf{r}}\right)\right)^{2}\right\rangle^{[\lambda, \beta]}>0
\end{gathered}
$$

which is always a positive quantity for all $\left(v_{\mathbf{r}}, u_{\mathbf{r}}\right) \neq 0$. A particular concern may arise with the following vector $\left(v_{\mathbf{r}}, u_{\mathbf{r}}\right)$ $=(1,1)$, for which

$$
\begin{aligned}
& \int d \mathbf{r} v_{\mathbf{r}} \delta n_{\mathbf{r}}=N-\langle N\rangle^{[\lambda, \beta]}, \\
& \int d \mathbf{r} u_{\mathbf{r}} \delta e_{\mathbf{r}}=H_{N}(z)-\left\langle H_{N}\right\rangle^{[\lambda, \beta]} .
\end{aligned}
$$

Should the average be made with the canonical ${ }^{39}$ or microcanonical ensemble, instead of the relevant ensemble (40), we would have $N-\langle N\rangle^{\text {can }}=0$ for example. In this case, the covariance matrix would have been positive semi-definite because it would have an eigenvector with null eigenvalue. ${ }^{39}$ In that case, there would be no inverse and would not be possible to ensure a one-to-one connection between the fields $n(\mathbf{r})$, $e(\mathbf{r})$ and $\lambda(\mathbf{r}), \beta(\mathbf{r})$. Nevertheless, even in this singular case, it is possible to show that a one-to-one connection exists in the non-singular subspace characterized by normalized fields satisfying

$$
\begin{gathered}
\int d \mathbf{r} n(\mathbf{r})=\bar{N}, \\
\int d \mathbf{r} e(\mathbf{r})=\bar{E},
\end{gathered}
$$

and normalized conjugate fields

$$
\begin{aligned}
& \int d \mathbf{r} \alpha(\mathbf{r})=\bar{\alpha}, \\
& \int d \mathbf{r} \beta(\mathbf{r})=\bar{\beta} .
\end{aligned}
$$

That is, for every $n, e$ satisfying (47) there is one and only one $\alpha, \beta$ satisfying (48). Obviously, we do not have to deal with this subtlety because the averages are performed with the "macrocanonical" relevant ensemble (40) for which the terms (46) do not vanish. We have then a one-to-one connection between $n(\mathbf{r}), e(\mathbf{r})$ and $\lambda(\mathbf{r}), \beta(\mathbf{r})$.

The entropy at the functional thermodynamics level is defined as the result of evaluating the entropy functional (12) at the relevant ensemble (40). The resulting entropy functional of the functional thermodynamics level of description is

$$
S_{\mathrm{FTD}}[n, e]=-\Omega_{\mathrm{FTD}}[\lambda, \beta]+\int d \mathbf{r}[\lambda(\mathbf{r}) n(\mathbf{r})+\beta(\mathbf{r}) e(\mathbf{r})]
$$

Here, $\lambda, \beta$ should be understood as functionals of $n, e$. The functional derivatives of the FTD entropy functional satisfy the following relationships:

$$
\begin{aligned}
& \frac{\delta S_{\mathrm{FTD}}[n, e]}{\delta n(\mathbf{r})}=\lambda(\mathbf{r}), \\
& \frac{\delta S_{\mathrm{FTD}}[n, e]}{\delta e(\mathbf{r})}=\beta(\mathbf{r}),
\end{aligned}
$$

and are given precisely by the conjugate variables.

\section{A. The temperature}

At this point, it proves convenient to perform the momenta integrals in the partition function (41), with the result

$$
\begin{aligned}
Z_{\mathrm{FTD}}[\lambda, \beta]= & \sum_{N=0}^{\infty} \frac{1}{N !} \int \prod_{i=0}^{N} \frac{d \mathbf{r}_{i}}{\Lambda^{3}\left(\mathbf{r}_{i}\right)} \\
& \times \exp \left\{-\sum_{j=1}^{N}\left[\lambda\left(\mathbf{r}_{j}\right)+\beta\left(\mathbf{r}_{j}\right) \phi_{j}\right]\right\},
\end{aligned}
$$

where the local thermal wavelength is defined as

$$
\Lambda(\mathbf{r}) \equiv\left(\frac{h^{2} \beta(\mathbf{r})}{2 m \pi}\right)^{\frac{1}{2}} .
$$

The functional derivative in (42) now becomes

$$
e(\mathbf{r})=\frac{3 n(\mathbf{r})}{2 \beta(\mathbf{r})}+\phi(\mathbf{r})
$$

where we have introduced the average potential energy field as

$$
\phi(\mathbf{r})=\operatorname{Tr}\left[\bar{\rho} \sum_{i=0}^{N} \phi_{i} \delta\left(\mathbf{r}-\mathbf{r}_{i}\right)\right],
$$

and $\phi_{i}=\frac{1}{2} \sum_{j \neq i} \phi\left(r_{i j}\right)+V^{\text {ext }}\left(\mathbf{r}_{i}\right)$ is the potential energy of particle $i$. It is natural to define the average kinetic energy field as $k(\mathbf{r})=e(\mathbf{r})-\phi(\mathbf{r})$ in such a way that the physical meaning of the Lagrange multiplier $\beta(\mathbf{r})$ becomes transparent as proportional to the inverse of the kinetic energy field. Note that we may introduce the microscopic kinetic density field as

$$
\hat{k}_{\mathbf{r}}(z)=\sum_{i=1}^{N} \frac{\mathbf{p}_{i}^{2}}{2 m_{i}} \delta\left(\mathbf{r}-\mathbf{r}_{i}\right)
$$

and, therefore,

$$
k(\mathbf{r})=\left\langle\hat{k}_{\mathbf{r}}\right\rangle^{\lambda, \beta}=\frac{3 n(\mathbf{r})}{2 \beta(\mathbf{r})},
$$

where $\langle\cdot\rangle^{\lambda, \beta}$ is an average performed with the relevant ensemble (40). By defining the temperature field $T(\mathbf{r}, t)$ as $2 / 3$ of the kinetic energy per particle divided by the Boltzmann constant, we have the interpretation of the field $\beta(\mathbf{r}, t)$ as proportional to the inverse of the temperature, that is, $\beta(\mathbf{r}, t)$ $=1 / k_{B} T(\mathbf{r})$.

\section{DENSITY FUNCTIONAL THEORY}

We would like to relate the FTD level of description just introduced with the DFT level of description. To this end we 
formulate in this section DFT within the framework of the maximum entropy principle, which is a slightly different presentation from usual ones. ${ }^{13,40}$ The level of description of DFT is characterized by the density field and the total energy, which are the averages of the following phase functions:

$$
\begin{aligned}
\hat{n}_{\mathbf{r}}(z) & =\sum_{i=1}^{N} \delta\left(\mathbf{r}-\mathbf{r}_{i}\right), \\
H_{N}(z) & =\sum_{i=0}^{N} \frac{\mathbf{p}_{i}^{2}}{2 m_{i}}+U_{N}\left(z_{N}\right) .
\end{aligned}
$$

The potential of interaction $U_{N}$ between the particles includes any external field $V_{\mathrm{ext}}(\mathbf{r})$. The averages of the macrostate variables will be denoted with $n(\mathbf{r}), E$ and these are assumed to be given.

The conjugate variables corresponding to $n(\mathbf{r}), E$ are $\lambda(\mathbf{r})$, $\beta$. The relevant ensemble (5) at the DFT level of description takes the form

$$
\bar{\rho}_{N}(z)=\frac{\rho^{0}}{Z_{D F T}[\lambda,(\beta)]} \exp \left\{-\beta H_{N}(z)-\int d \mathbf{r} \lambda(\mathbf{r}) \hat{n}_{\mathbf{r}}(z)\right\},
$$

where we have introduced the partition function of the DFT level of description as

$$
Z_{D F T}[\lambda,(\beta)]=\sum_{N=0}^{\infty} \int d z_{N} \rho^{0} \exp \left\{-\beta H_{N}-\sum_{i=1}^{N} \lambda\left(\mathbf{r}_{i}\right)\right\} .
$$

By its very structure, the conjugate variable $\lambda(\mathbf{r})$ could be interpreted as an additional (adimensionalized) external field, but we prefer to distinguish the nature of the Lagrange multiplier from the external potential because for other variables different from the density, the conjugate variable cannot be simply interpreted as an external potential. Note that if the Hamiltonian dynamics contains the external potential, then the relevant ensemble (58) is not the equilibrium ensemble of the system, which is only recovered when the Lagrange multiplier $\lambda(\mathbf{r})$ is constant.

The partition function (59) is a functional of the conjugate variable $\lambda(\mathbf{r})$ and a function of $\beta$. The notation $Z_{D F T}[\lambda,(\beta)]$ emphasizes the functional dependence with a square bracket, whereas the parenthesis reminds us that $\beta$ is a scalar, not a function.

The connection (8) between the conjugate variables and the given averages is now

$$
\begin{aligned}
& \frac{\delta \Omega_{\mathrm{DFT}}[\lambda,(\beta)]}{\delta \lambda(\mathbf{r})}=n(\mathbf{r}), \\
& \frac{\partial \Omega_{\mathrm{DFT}}[\lambda,(\beta)]}{\partial \beta}=E,
\end{aligned}
$$

where the dimensionless potential at the DFT level is

$$
\Omega_{\mathrm{DFT}}[\lambda,(\beta)] \equiv-\ln Z_{D F T}[\lambda,(\beta)] .
$$

The first equation in Eqs. (60) involves the functional derivative with respect to the field $\lambda(\mathbf{r})$, whereas the second equation involves a usual partial derivative. One way to solve these equations is by assuming in the first equation that $\beta$ is a parameter allowing to solve $\lambda(\mathbf{r})$ as a functional of $n(\mathbf{r})$ that depends parametrically on $\beta$. Substituting this functional into the second equation, we obtain an implicit equation between $\beta$ and $E$.

Finally, the entropy of the DFT level of description is obtained after inserting the relevant ensemble (58) into the Gibbs entropy functional (12) with the result

$$
S_{\mathrm{DFT}}[n,(E)]=-\Omega_{\mathrm{DFT}}[\lambda,(\beta)]+\int d \mathbf{r} \lambda(\mathbf{r}) n(\mathbf{r})+\beta E .
$$

It is natural to define a dimensionless Helmholtz potential according to

$$
F_{\mathrm{DFT}}[n,(\beta)] \equiv \Omega[\lambda,(\beta)]-\int d \mathbf{r} \lambda(\mathbf{r}) n(\mathbf{r}),
$$

where in the last equation $\lambda(\mathbf{r})$ should be understood as a functional of $n(\mathbf{r})$ and a function of $\beta$, i.e., $\lambda[n,(\beta)](\mathbf{r})$. The functional (63) is the usual free energy functional of DFT up to factors of $\beta^{-1}=k_{B} T$.

The DFT level of description can be obtained from the functional thermodynamics level of description because the $\mathrm{CG}$ variables of the former can be obtained as linear combinations of the fine grained variables $n_{\mathbf{r}}(z), e_{\mathbf{r}}(z)$. Therefore, the bridge theorem (23) applies and, consequently, if we know the explicit form of the entropy functional (49) at the FTD level of description, we know the entropy functional (62) at the DFT level of description. The entropy of the DFT level of description can be obtained according to the bridge theorem (23) from the entropy of the FTD level by maximizing the last one subject to give the restrictions

$$
\begin{aligned}
n(\mathbf{r}) & =n_{0}(\mathbf{r}), \\
\int d \mathbf{r} e(\mathbf{r}) & =E .
\end{aligned}
$$

The unconstrained functional to maximize is now

$$
S_{\mathrm{FTD}}[n, e]-\int d \mathbf{r} \lambda(\mathbf{r}) n(\mathbf{r})-\beta_{0} \int d \mathbf{r} e(\mathbf{r}) .
$$

The maximum occurs at $n^{*}(\mathbf{r}), e^{*}(\mathbf{r})$, which are the solutions of

$$
\begin{aligned}
& \frac{\delta S_{\mathrm{FTD}}\left[n^{*}(\mathbf{r}), e^{*}(\mathbf{r})\right]}{\delta n(\mathbf{r})}=\lambda(\mathbf{r}), \\
& \frac{\delta S_{\mathrm{FTD}}\left[n^{*}(\mathbf{r}), e^{*}(\mathbf{r})\right]}{\delta e(\mathbf{r})}=\beta_{0} .
\end{aligned}
$$

The profiles $n^{*}(\mathbf{r}), e^{*}(\mathbf{r})$ depend on $\lambda(\mathbf{r}), \beta_{0}$ and, after substitution into the constraints (64), we obtain the dependence of $\lambda(\mathbf{r}), \beta_{0}$ on $n_{0}(\mathbf{r}), E$. Therefore, we have the profiles $n^{*}(\mathbf{r})$, $e^{*}(\mathbf{r})$ as functions of $n_{0}(\mathbf{r}), E$, that is,

$$
\begin{aligned}
& n^{*}(\mathbf{r})=n_{0}(\mathbf{r}), \\
& e^{*}(\mathbf{r})=e^{*}(\mathbf{r})\left[n_{0}(\mathbf{r}),(E)\right] .
\end{aligned}
$$

According to the bridge theorem (23), substitution of (67) into the FTD entropy leads to the DFT entropy, that is,

$$
S_{\mathrm{DFT}}\left[n_{0}(\mathbf{r}),(E)\right]=S_{\mathrm{FTD}}\left(n_{0}(\mathbf{r}), e^{*}(\mathbf{r})\left[n_{0}(\mathbf{r}),(E)\right]\right) .
$$


This equation gives the explicit relationship between the entropies of the two levels of description. Note that by comparing (59) and (41) we arrive at the following relationship between the dimensionless grand potentials (61) and (43):

$$
\Omega_{\mathrm{DFT}}\left[\lambda(\mathbf{r}),\left(\beta_{0}\right)\right]=\Omega_{\mathrm{FTD}}\left[\lambda(\mathbf{r}), \beta_{0}\right] .
$$

That is, the dimensionless grand potential at the DFT level is obtained from the dimensionless grand potential at the FTD level by simply setting the Lagrange multiplier to a constant $\beta(\mathbf{r})=\beta_{0}$ in the latter.

\section{DYNAMIC TRANSPORT EQUATIONS FOR FTD}

We now particularize the general dynamic equation (33) to the case that the relevant variables are the number and energy density fields, which are assumed to be the only slow variables in the system. Microscopically these variables are defined in Eq. (37). The averages of these phase functions with respect to the solution $\rho_{t}(z)$ of the Liouville equation are denoted by

$$
\begin{aligned}
& n(\mathbf{r}, t)=\operatorname{Tr}\left[\rho_{t} \hat{n}_{\mathbf{r}}\right], \\
& e(\mathbf{r}, t)=\operatorname{Tr}\left[\rho_{t} \hat{e}_{\mathbf{r}}\right] .
\end{aligned}
$$

The time derivatives of the relevant variables, i.e., the terms $i L A_{i}$ appearing in Eqs. (32) and (34), now become

$$
\begin{aligned}
i L \hat{n}_{\mathbf{r}}(z)= & -\sum_{i} \mathbf{v}_{i} \nabla \delta\left(\mathbf{r}-\mathbf{r}_{i}\right), \\
i L \hat{e}_{\mathbf{r}}(z)= & -\sum_{i} \mathbf{v}_{i} e_{i} \nabla \delta\left(\mathbf{r}-\mathbf{r}_{i}\right) \\
& +\frac{1}{4} \sum_{i j} \mathbf{F}_{i j}\left(\mathbf{v}_{i}+\mathbf{v}_{j}\right)\left[\delta\left(\mathbf{r}-\mathbf{r}_{i}\right)-\delta\left(\mathbf{r}-\mathbf{r}_{j}\right)\right] .
\end{aligned}
$$

By using the usual trick ${ }^{32}$

$$
\delta\left(\mathbf{r}-\mathbf{r}_{i}\right)-\delta\left(\mathbf{r}-\mathbf{r}_{j}\right)=-\nabla \int_{0}^{1} d \epsilon \mathbf{r}_{i j} \delta\left(\mathbf{r}-\mathbf{r}_{j}-\epsilon \mathbf{r}_{i j}\right),
$$

we may express the time derivatives as divergences of fluxes, that is,

$$
\begin{aligned}
i L \hat{n}_{\mathbf{r}}(z) & =-\nabla \hat{\mathbf{J}}_{\mathbf{r}}(z), \\
i L \hat{e}_{\mathbf{r}}(z) & =-\nabla \hat{\mathbf{Q}}_{\mathbf{r}}(z),
\end{aligned}
$$

where the current $\hat{\mathbf{J}}_{\mathbf{r}}$ and heat flux $\hat{\mathbf{Q}}_{\mathbf{r}}$ have the usual microscopic expressions

$$
\begin{aligned}
\hat{\mathbf{J}}_{\mathbf{r}}(z)= & \sum_{i} \mathbf{v}_{i} \delta\left(\mathbf{r}-\mathbf{r}_{i}\right), \\
\hat{\mathbf{Q}}_{\mathbf{r}}(z)= & \sum_{i} \mathbf{v}_{i} e_{i} \delta\left(\mathbf{r}-\mathbf{r}_{i}\right) \\
& +\frac{1}{4} \sum_{i j} \mathbf{F}_{i j}\left(\mathbf{v}_{i}+\mathbf{v}_{j}\right) \int_{0}^{1} d \epsilon \mathbf{r}_{i j} \delta\left(\mathbf{r}-\mathbf{r}_{j}-\epsilon \mathbf{r}_{i j}\right) .
\end{aligned}
$$

Now, we consider the reversible part $v_{i}(t)$ in Eq. (32), which takes the form

$$
\begin{aligned}
& \operatorname{Tr}\left[\bar{\rho}_{t} i L \hat{n}_{\mathbf{r}}\right]=0, \\
& \operatorname{Tr}\left[\bar{\rho}_{t} i L \hat{e}_{\mathbf{r}}\right]=0,
\end{aligned}
$$

because both terms include a Gaussian momentum integral of an even power of the velocity. In the irreversible part, for the same reason, the projected currents (35) are simply

$$
\begin{aligned}
& \mathcal{Q}_{t} i L \hat{n}_{\mathbf{r}}=-\nabla \cdot \hat{\mathbf{J}}_{\mathbf{r}}(z), \\
& \mathcal{Q}_{t} i L \hat{e}_{\mathbf{r}}=-\nabla \cdot \hat{\mathbf{Q}}_{\mathbf{r}}(z) .
\end{aligned}
$$

The dissipative matrix in Eq. (34) becomes now of the form $D_{i j} \rightarrow \nabla_{\mathbf{r}} \nabla_{\mathbf{r}^{\prime}} \mathbf{M}_{\mathbf{r}, \mathbf{r}^{\prime}}$ with

$$
\begin{aligned}
& \nabla_{\mathbf{r}} \nabla_{\mathbf{r}^{\prime}}\left(\begin{array}{ll}
\mathbf{M}_{\mathbf{r r}}^{\mathrm{J}} & \mathbf{M}_{\mathbf{r} \mathbf{r}^{\prime}}^{J Q} \\
\mathbf{M}_{\mathbf{r r}}^{Q J} & \mathbf{M}_{\mathbf{r r}}^{Q Q}
\end{array}\right) \\
& =\nabla_{\mathbf{r}} \nabla_{\mathbf{r}^{\prime}}\left(\begin{array}{l}
\int_{0}^{\infty} d t^{\prime} \operatorname{Tr}\left[\bar{\rho}_{t} \hat{\mathbf{J}}_{\mathbf{r}^{\prime}} \hat{\mathbf{J}}_{\mathbf{r}}\left(t^{\prime}\right)\right] \int_{0}^{\infty} d t^{\prime} \operatorname{Tr}\left[\bar{\rho}_{t} \hat{\mathbf{Q}}_{\mathbf{r}^{\prime}} \hat{\mathbf{J}}_{\mathbf{r}}\left(t^{\prime}\right)\right] \\
\int_{0}^{\infty} d t^{\prime} \operatorname{Tr}\left[\bar{\rho}_{t} \hat{\mathbf{J}}_{\mathbf{r}^{\prime}} \hat{\mathbf{Q}}_{\mathbf{r}}\left(t^{\prime}\right)\right] \int_{0}^{\infty} d t^{\prime} \operatorname{Tr}\left[\bar{\rho}_{t} \hat{\mathbf{Q}}_{\mathbf{r}^{\prime}} \hat{\mathbf{Q}}_{\mathbf{r}}\left(t^{\prime}\right)\right]
\end{array}\right) .
\end{aligned}
$$

The final dynamic equations (33) take in the FTD level of description the form

$$
\begin{aligned}
\partial_{t} n(\mathbf{r}, t)= & -\nabla_{\mathbf{r}} \int d \mathbf{r}^{\prime} \mathbf{M}_{\mathbf{r} \mathbf{r}^{\prime}}^{J J} \nabla_{\mathbf{r}^{\prime}} \frac{\delta S}{\delta n\left(\mathbf{r}^{\prime}, t\right)} \\
& -\nabla_{\mathbf{r}} \int d \mathbf{r}^{\prime} \mathbf{M}_{\mathbf{r r}^{\prime}}^{J Q} \nabla_{\mathbf{r}^{\prime}} \frac{\delta S}{\delta e\left(\mathbf{r}^{\prime}, t\right)}, \\
\partial_{t} e(\mathbf{r}, t)= & -\nabla_{\mathbf{r}} \int d \mathbf{r}^{\prime} \mathbf{M}_{\mathbf{r} \mathbf{r}^{\prime}}^{Q J} \nabla_{\mathbf{r}^{\prime}} \frac{\delta S}{\delta n\left(\mathbf{r}^{\prime}, t\right)} \\
& -\nabla_{\mathbf{r}} \int d \mathbf{r}^{\prime} \mathbf{M}_{\mathbf{r r}^{\prime}}^{Q Q} \nabla_{\mathbf{r}^{\prime}} \frac{\delta S}{\delta e\left(\mathbf{r}^{\prime}, t\right)},
\end{aligned}
$$

where partial integrations have been performed. The nonlocal hydrodynamic equations (78) are one of the main results of the paper. These equations, being in divergence form, conserve the total number of particles and total energy defined as

$$
\begin{aligned}
N & =\int d \mathbf{r} n(\mathbf{r}), \\
E & =\int d \mathbf{r} e(\mathbf{r}) .
\end{aligned}
$$

At the same time, they have as a Lyapunov functional the entropy functional (49), a property that is inherited from the general property (36) and that can be explicitly checked. Equations (78) describe the tendency towards equilibrium in an isolated system.

Of course, in order for these equations to be useful, it is necessary to have explicit expressions for the entropy functional $S[n, e]$ and for the dissipative matrix of transport coefficients $\mathbf{M}_{\mathbf{r}, \mathbf{r}^{\prime}}$. The latter is given in Eq. (77) in terms of GreenKubo expressions involving correlations (computed with the relevant ensemble and, therefore, dependent functionally on $n, e)$ of the microscopic fluxes. In Secs. VI-VII, we present several models for these quantities. 


\section{MODELS FOR THE FTD ENTROPY}

The functional form of the entropy at the FTD level contains all the information about the equilibrium profiles of number and energy densities and also of the static correlations of these variables. In order to obtain these quantities it is necessary to have explicit models for the entropy functional. In DFT, different approaches have been considered in order to produce suitable functional models ranging from the Local Density Approximation (LDA), the Square Gradient Approximation (SGA), ${ }^{41}$ the Weighted Density Approximation (WDA), ${ }^{42}$ Taylor expansions around a reference system ${ }^{43}$ up to the modern models based on the fundamental measure theory. ${ }^{17,44}$ While similar schemes may be developed at the FTD level, we restrict in the present work to three simple models, the ideal gas, the hard sphere fluid, and the local model.

\section{A. Ideal gas}

In the case of an ideal gas, the grand partition function, Eq. (41), can be explicitly computed:

$$
\begin{aligned}
Z_{F T D}^{\mathrm{id}}[\lambda, \beta]= & \sum_{N=0}^{\infty} \frac{1}{N ! h^{3 N}} \\
& \times\left[\int d \mathbf{r}\left(\frac{2 m \pi}{\beta(\mathbf{r})}\right)^{\frac{3}{2}} \exp \{-\lambda(\mathbf{r})-\beta(\mathbf{r}) V(\mathbf{r})\}\right]^{N} \\
= & \exp \left\{\int \frac{d \mathbf{r}}{\Lambda^{3}(\mathbf{r})} \exp \{-\lambda(\mathbf{r})-\beta(\mathbf{r}) V(\mathbf{r})\}\right\}
\end{aligned}
$$

where the local thermal wavelength is defined in Eq. (52). The dimensionless grand potential is

$$
\Omega_{F T D}^{\mathrm{id}}[\lambda, \beta]=-\int \frac{d \mathbf{r}}{\Lambda^{3}(\mathbf{r})} \exp \{-\lambda(\mathbf{r})-\beta(\mathbf{r}) V(\mathbf{r})\} .
$$

The functional derivatives with respect to $\lambda$ and $\beta$ are

$$
\begin{aligned}
\frac{\delta \Omega_{F T D}^{\mathrm{id}}[\lambda, \beta]}{\delta \lambda(\mathbf{r})}= & \frac{1}{\Lambda^{3}(\mathbf{r})} \exp \{-\lambda(\mathbf{r})-\beta(\mathbf{r}) V(\mathbf{r})\}, \\
\frac{\delta \Omega_{F T D}^{\mathrm{id}}[\lambda, \beta]}{\delta \beta(\mathbf{r})}= & \frac{1}{\Lambda^{3}(\mathbf{r})} \exp \{-\lambda(\mathbf{r})-\beta(\mathbf{r}) V(\mathbf{r})\} \\
& \times\left[\frac{3}{2 \beta(\mathbf{r})}+V(\mathbf{r})\right] .
\end{aligned}
$$

Because of (42), we have

$$
\begin{aligned}
\frac{1}{\Lambda^{3}(\mathbf{r})} \exp \{-\lambda(\mathbf{r})-\beta(\mathbf{r}) V(\mathbf{r})\} & =n(\mathbf{r}), \\
\frac{3 n(\mathbf{r})}{2 \beta(\mathbf{r})}+n(\mathbf{r}) V(\mathbf{r}) & =e(\mathbf{r}) .
\end{aligned}
$$

Therefore, we may obtain the conjugate variables in explicit form as function of the relevant variables:

$$
\begin{aligned}
& \beta(\mathbf{r})=\frac{3}{2} \frac{n(\mathbf{r})}{e(\mathbf{r})-n(\mathbf{r}) V(\mathbf{r})}, \\
& \lambda(\mathbf{r})=-\frac{3}{2} \frac{n(\mathbf{r}) V(\mathbf{r})}{e(\mathbf{r})-n(\mathbf{r}) V(\mathbf{r})}-\ln \left[\Lambda^{3}(\mathbf{r}) n(\mathbf{r})\right] .
\end{aligned}
$$

With these results we may now simply compute the entropy (49) for the ideal gas, with the final result

$$
S_{F T D}^{\mathrm{id}}[n, e]=-\int d \mathbf{r} n(\mathbf{r})\left[\ln \left[\Lambda^{3}(\mathbf{r}) n(\mathbf{r})\right]-\frac{5}{2}\right] .
$$

Here, the thermal wavelength is a function of the fields $n, e$ through $\beta$ (i.e., Eq. (52)).

We may use the bridge theorem in order to obtain the entropy of the MT level of description. By maximizing (85) subject to the constraints that $n(\mathbf{r}), e(\mathbf{r})$ give prescribed values $N, E$, we obtain a homogeneous solution $n^{*}(\mathbf{r})=n$ y $e^{*}(\mathbf{r})$ $=e$, which implies $\beta(\mathbf{r})=\beta_{0}$. By evaluating the entropy functional (85) at these equilibrium solutions, we obtain the wellknown Sackur-Tetrode entropy for the ideal gas:

$$
S_{\mathrm{MT}}^{\mathrm{id}}(N, E)=N \ln \left[\frac{V}{N \Lambda^{3}}\right]+\frac{5}{2} N .
$$

\section{B. Hard spheres}

A system of hard spheres is characterized by the fact that its total energy is fully kinetic, a fact also referred as the hard sphere system being athermal. There are many good approximations for the hard sphere free energy functional at the DFT level. ${ }^{41,44}$ For this reason, we would like to use this DFT information in order to formulate the entropy functional of a hard sphere system at the level of FTD. This is actually possible precisely because the system is athermal.

In DFT we have from (59), after integrating over momenta:

$$
\begin{aligned}
& Z_{D F T}^{\mathrm{hs}}\left[\lambda,\left(\beta_{0}\right)\right] \\
& =\sum_{N=0}^{\infty} \frac{1}{N !} \int \prod_{i}^{N} d \mathbf{r}_{i}\left(\frac{2 m \pi h^{2}}{\beta_{0}}\right)^{\frac{3}{2}} \\
& \quad \times \exp \left\{-\sum_{i=1}^{N}\left(\lambda\left(\mathbf{r}_{i}\right)+\beta_{0} V\left(\mathbf{r}_{i}\right)\right)\right\} f\left(\mathbf{r}_{1}, \cdots, \mathbf{r}_{N}\right),
\end{aligned}
$$

whereas in FTD we have from (41), after integrating over momenta:

$$
\begin{aligned}
& Z_{F T D}^{\mathrm{hs}}[\lambda, \beta] \\
& =\sum_{N=0}^{\infty} \frac{1}{N !} \int \prod_{i}^{N} d \mathbf{r}_{i}\left(\frac{2 m \pi h^{2}}{\beta\left(\mathbf{r}_{i}\right)}\right)^{\frac{3}{2}} \\
& \quad \times \exp \left\{-\sum_{i=1}^{N}\left(\lambda\left(\mathbf{r}_{i}\right)+\beta\left(\mathbf{r}_{i}\right) V\left(\mathbf{r}_{i}\right)\right)\right\} f\left(\mathbf{r}_{1}, \cdots, \mathbf{r}_{N}\right),
\end{aligned}
$$


where we have introduced the function (related to the Meyer function):

$$
f\left(\mathbf{r}_{1}, \ldots, \mathbf{r}_{N}\right) \equiv \exp \left\{-\sum_{i=1}^{N} \beta\left(\mathbf{r}_{i}\right) \frac{1}{2} \sum_{j} \phi_{i j}^{\mathrm{hs}}\right\} .
$$

It is obvious that (87) is obtained from (88) by taking in the latter a constant value for the field $\beta(\mathbf{r})=\beta_{0}$. In the above expressions, $\phi_{i j}^{\mathrm{hs}}$ is the hard sphere potential for particles $i, j$. This potential is zero if the spheres do not overlap, and infinite if they overlap. As a consequence, $f\left(\mathbf{r}_{1}, \ldots, \mathbf{r}_{N}\right)$ is zero if any two spheres overlap and 1 otherwise, irrespective of the value of $\beta(\boldsymbol{r})$. Therefore, Eq. (88) can be written as

$$
\begin{aligned}
& Z_{F T D}^{\mathrm{hs}}[\lambda, \beta] \\
& =\sum_{N=0}^{\infty} \frac{1}{N !} \int \prod_{i}^{N} d \mathbf{r}_{i}\left(\frac{2 m \pi h^{2}}{\beta_{0}}\right)^{\frac{3}{2}} \\
& \quad \times \exp \left\{-\sum_{i=1}^{N}\left(\lambda\left(\mathbf{r}_{i}\right)+\beta\left(\mathbf{r}_{i}\right) V\left(\mathbf{r}_{i}\right)+\frac{3}{2} \ln \frac{\beta\left(\mathbf{r}_{i}\right)}{\beta_{0}}\right)\right\} \\
& \quad \times f\left(\mathbf{r}_{1}, \ldots, \mathbf{r}_{N}\right) \\
& =Z_{D F T}^{\mathrm{hs}}\left[\lambda+\left(\beta-\beta_{0}\right) V+\frac{3}{2} \ln \frac{\beta}{\beta_{0}},\left(\beta_{0}\right)\right]
\end{aligned}
$$

This expression is valid for any $\beta_{0}$. Therefore, if we know the functional form of $Z_{D F T}^{\mathrm{hs}}\left[\lambda,\left(\beta_{0}\right)\right]$ for a hard sphere system at the DFT level of description, then we automatically know the functional form of $Z_{F T D}^{\mathrm{hs}}[\lambda, \beta]$ at the FTD level. The dimensionless grand canonical potential at both levels, DFT and FTD, is also related as

$$
\Omega_{\mathrm{FTD}}^{\mathrm{hs}}[\lambda, \beta]=\Omega_{\mathrm{DFT}}^{\mathrm{hs}}\left[\lambda+\left(\beta-\beta_{0}\right) V+\frac{3}{2} \ln \frac{\beta}{\beta_{0}},\left(\beta_{0}\right)\right] .
$$

Now, from Eqs. (42), we readily obtain the result:

$$
\begin{aligned}
& n(\mathbf{r})=\frac{\delta \Omega_{D F T}^{\mathrm{hs}}}{\delta \lambda(\mathbf{r})}\left[\lambda+\left(\beta-\beta_{0}\right) V+\frac{3}{2} \ln \frac{\beta}{\beta_{0}},\left(\beta_{0}\right)\right], \\
& e(\mathbf{r})=\frac{3 n(\mathbf{r})}{2 \beta(\mathbf{r})}+n(\mathbf{r}) V(\mathbf{r}) .
\end{aligned}
$$

The last identity is a reflection that the hard sphere system has only kinetic energy.

Let us assume that we know the functional form of the free energy density functional $F_{D F T}^{\mathrm{hs}}[n,(\beta)]$ introduced in (63) for the case of a hard sphere. We may take the functional derivative,

$$
\frac{\delta F_{D F T}^{\mathrm{hs}}[n,(\beta)]}{\delta n(\mathbf{r})}=\lambda[n,(\beta)](\mathbf{r}),
$$

that can be formally inverted in order to get $n[\lambda,(\beta)](\mathbf{r})$ as a functional of $\lambda(\mathbf{r})$. With this input information, we can obtain the grand potential through Eq. (63) as

$\Omega_{D F T}^{\mathrm{hs}}[\lambda,(\beta)] \equiv F_{D F T}^{\mathrm{hs}}[n[\lambda,(\beta)],(\beta)]+\int d \mathbf{r} \lambda(\mathbf{r}) n[\lambda,(\beta)](\mathbf{r})$.
Then, Eq. (91) allows us to obtain

$$
\begin{aligned}
\Omega_{\mathrm{FTD}}^{\mathrm{hs}}[\lambda, \beta]= & \Omega_{\mathrm{DFT}}^{\mathrm{hs}}\left[\lambda+\left(\beta-\beta_{0}\right) V+\frac{3}{2} \ln \frac{\beta}{\beta_{0}},\left(\beta_{0}\right)\right] \\
= & F_{D F T}\left[n\left[\lambda+\left(\beta-\beta_{0}\right) V+\frac{3}{2} \ln \frac{\beta}{\beta_{0}}\right],\left(\beta_{0}\right)\right] \\
& +\int d \mathbf{r}\left(\lambda(\mathbf{r})+\left(\beta-\beta_{0}\right) V+\frac{3}{2} \ln \frac{\beta(\mathbf{r})}{\beta_{0}}\right) \\
& \times n\left[\lambda+\left(\beta-\beta_{0}\right) V+\frac{3}{2} \ln \frac{\beta}{\beta_{0}},\left(\beta_{0}\right)\right](\mathbf{r}) .
\end{aligned}
$$

Finally, we need to take the Legendre transform of this in order to obtain the entropy functional according to Eq. (49), that is,

$$
\begin{aligned}
S_{\mathrm{FTD}}^{\mathrm{hs}}[n, e] & \\
= & -\Omega_{\mathrm{FTD}}^{\mathrm{hs}}[\lambda, \beta]+\int d \mathbf{r}[\lambda(\mathbf{r}) n(\mathbf{r})+\beta(\mathbf{r}) e(\mathbf{r})] \\
= & -F_{D F T}^{\mathrm{hs}}\left[n,\left(\beta_{0}\right)\right]-\int d \mathbf{r}\left(\lambda(\mathbf{r})+\left(\beta-\beta_{0}\right) V+\frac{3}{2} \ln \frac{\beta(\mathbf{r})}{\beta_{0}}\right) \\
& \times n(\mathbf{r})+\int d \mathbf{r}[\lambda(\mathbf{r}) n(\mathbf{r})+\beta(\mathbf{r}) e(\mathbf{r})] \\
= & -F_{D F T}^{\mathrm{hs}}\left[n,\left(\beta_{0}\right)\right]-\int d \mathbf{r} \frac{3}{2} n(\mathbf{r}) \ln \frac{\beta(\mathbf{r})}{\beta_{0}} \\
& -\int d \mathbf{r}\left(\beta(\mathbf{r})-\beta_{0}\right) V(\mathbf{r}) n(\mathbf{r})+\int d \mathbf{r} \beta(\mathbf{r}) e(\mathbf{r}) \\
= & -F_{D F T}^{\mathrm{hs}}\left[n,\left(\beta_{0}\right)\right]+\beta_{0} \int d \mathbf{r} V(\mathbf{r}) n(\mathbf{r}) \\
& -\int d \mathbf{r} \frac{3}{2} n(\mathbf{r})\left[\ln \frac{\beta(\mathbf{r})}{\beta_{0}}-1\right] .
\end{aligned}
$$

This gives the explicit exact form of the entropy functional $S_{\mathrm{FTD}}^{\mathrm{hs}}[n, e]$ of a hard sphere system, provided that the free energy functional $F_{D F T}^{\mathrm{hs}}\left[n,\left(\beta_{0}\right)\right]$ of the hard sphere is known. Note that in this expression, $F_{D F T}^{\mathrm{hs}}\left[n,\left(\beta_{0}\right)\right]$ is the free energy density in the presence of the external field, and, therefore,

$$
F_{D F T}^{0 \mathrm{hs}}\left[n,\left(\beta_{0}\right)\right] \equiv F_{D F T}^{\mathrm{hs}}\left[n,\left(\beta_{0}\right)\right]-\beta_{0} \int d \mathbf{r} V(\mathbf{r}) n(\mathbf{r})
$$

is the intrinsic (zero external field) free energy of the hard sphere system. Finally, we end up with the following remarkably simple and exact result for the entropy functional of the hard sphere system

$$
S_{\mathrm{FTD}}^{\mathrm{hs}}[n, e]=F_{D F T}^{0 \mathrm{hs}}\left[n,\left(\beta_{0}\right)\right]+\int d \mathbf{r} \frac{3}{2} n(\mathbf{r})\left[\ln \frac{\beta(\mathbf{r})}{\beta_{0}}-1\right],
$$

where $\beta(\mathbf{r})$ is, from (92), the following function of $n(\mathbf{r}), e(\mathbf{r})$ :

$$
\beta(\mathbf{r})=\frac{3}{2} \frac{n(\mathbf{r})}{e(\mathbf{r})-n(\mathbf{r}) V(\mathbf{r})},
$$

and $\beta_{0}$ is arbitrary. One check that we may perform on this expression is the ideal gas limit. In the limit in which the radius of the hard sphere vanishes, the free energy $F_{D F T}^{0 \mathrm{hs}}\left[n,\left(\beta_{0}\right)\right]$ of 
the hard sphere is just the dimensionless free energy of the ideal gas, which is

$$
F_{D F T}^{\mathrm{id}}\left[n,\left(\beta_{0}\right)\right]=\int d \mathbf{r} n(\mathbf{r})\left[\ln \Lambda_{0}^{3} n(\mathbf{r})-1\right] .
$$

If we substitute this free energy functional into (98), we end up with the correct ideal gas result (85) for the entropy functional.

\section{Local model}

We consider finally a local model for the entropy functional, which is of the form

$$
S_{\mathrm{FTD}}[n, e]=\int d \mathbf{r} s(n(\mathbf{r}), e(\mathbf{r})),
$$

where the entropy density $s(n, e)$ is an a priori unknown function. We may use, however, the bridge theorem (23) and compute the entropy of the macroscopic thermodynamics level of description. By maximizing (101) subject to the restrictions

$$
\begin{aligned}
& \int d \mathbf{r} n(\mathbf{r})=N, \\
& \int d \mathbf{r} e(\mathbf{r})=E,
\end{aligned}
$$

we obtain the equations

$$
\begin{aligned}
& \frac{\partial s}{\partial n}(n(\mathbf{r}), e(\mathbf{r}))=\lambda_{0}, \\
& \frac{\partial s}{\partial n}(n(\mathbf{r}), e(\mathbf{r}))=\beta_{0},
\end{aligned}
$$

where $\lambda_{0}, \beta_{0}$ are the Lagrange multipliers enforcing (102). The solution of (103) is $n^{*}(\mathbf{r}), n^{*}(\mathbf{r})=n_{0}, e^{*}(\mathbf{r})=e_{0}$. The constants $n_{0}, e_{0}$ are obtained by the requirement that the fields $n^{*}(\mathbf{r})=n_{0}, e^{*}(\mathbf{r})$ satisfy (102), giving $n_{0}=N / V$ and $e_{0}=E / V$, where $V$ is the volume of the system. The entropy at the macroscopic thermodynamics level is then, according to the bridge theorem (23),

$$
\begin{aligned}
S_{\mathrm{MT}}(N, E) & =S_{\mathrm{FTD}}\left[n^{*}, e^{*}\right] \\
& =V s\left(n_{0}, e_{0}\right) .
\end{aligned}
$$

Therefore, we obtain that the function $s(n, e)$ is given by the entropy of the macroscopic thermodynamics level of description through

$$
s(n, e)=\frac{1}{V} S_{\mathrm{MT}}(V n, V e)
$$

\section{THE TRANSPORT COEFFICIENTS}

The transport coefficients introduced in (77) are given in terms of time integrals of correlations of fluxes, where the ensemble to be used is the relevant ensemble. Because the relevant ensemble is a functional of the fields $n(\mathbf{r}), e(\mathbf{r})$ through the conjugate variables $\lambda(\mathbf{r}), \beta(\mathbf{r})$, the transport coefficients inherit this functional dependence. This poses the problem of computing the transport coefficients in a molecular dynamics simulation. Indeed, what can be computed in MD are equilibrium time averages that sample the equilibrium ensemble (at constant $\lambda, \beta$ ). It is apparent that unless some approximations are taken, it is impossible to explore the functional space of the fields $n(\mathbf{r}), e(\mathbf{r})$ in order to obtain the functional dependence of the transport coefficients.

The main observation is that we expect that the correlations of $\hat{\mathbf{J}}_{\mathbf{r}}, \hat{\mathbf{Q}}_{\mathbf{r}}$ appearing in (77) decay in molecular length scales. In situations where the gradients of the conjugate fields $\lambda(\mathbf{r}), \beta(\mathbf{r})$ vary in length scales much larger than the molecular correlation lengths (as in near equilibrium situations), we may expect a local approximation for the evolution equations. Consider, for example, the first term in the density equation in (78). In a local approximation, we assume

$$
\int d \mathbf{r}^{\prime} \mathbf{M}_{\mathbf{r r}^{\prime}}^{J J} \nabla_{\mathbf{r}^{\prime}} \lambda\left(\mathbf{r}^{\prime}, t\right) \approx\left[\int d \mathbf{r}^{\prime} \mathbf{M}_{\mathbf{r r}^{\prime}}^{J J}\right] \nabla_{\mathbf{r}} \lambda(\mathbf{r}, t) .
$$

The approximation holds because in the molecular length scale of decay of the correlation of $\hat{\mathbf{J}}_{\mathbf{r}}$, the gradient of $\lambda(\mathbf{r})$ hardly changes.

Under this local approximation, Eqs. (78) take the local form

$$
\begin{aligned}
& \partial_{t} n(\mathbf{r}, t)=-\nabla D(\mathbf{r}, t) \nabla \frac{\delta S}{\delta n(\mathbf{r})}-\nabla C(\mathbf{r}, t) \nabla \frac{\delta S}{\delta e(\mathbf{r})}, \\
& \partial_{t} e(\mathbf{r}, t)=-\nabla C(\mathbf{r}, t) \nabla \frac{\delta S}{\delta n(\mathbf{r})}-\nabla K(\mathbf{r}, t) \nabla \frac{\delta S}{\delta e(\mathbf{r})} .
\end{aligned}
$$

The local transport coefficients are defined as

$$
\begin{aligned}
& D(\mathbf{r}, t)=\int d \mathbf{r}^{\prime} \mathbf{M}_{\mathbf{r} \mathbf{r}^{\prime}}^{J J}=\int_{0}^{\infty} d t^{\prime} \operatorname{Tr}\left[\bar{\rho}_{t} \hat{\mathbf{J}} \hat{\mathbf{J}}_{\mathbf{r}}\left(t^{\prime}\right)\right], \\
& C(\mathbf{r}, t)=\int d \mathbf{r}^{\prime} \mathbf{M}_{\mathbf{r} \mathbf{r}^{\prime}}^{J Q}=\int_{0}^{\infty} d t^{\prime} \operatorname{Tr}\left[\bar{\rho}_{t} \hat{\mathbf{J}} \hat{\mathbf{Q}}_{\mathbf{r}}\left(t^{\prime}\right)\right], \\
& K(\mathbf{r}, t)=\int d \mathbf{r}^{\prime} \mathbf{M}_{\mathbf{r} \mathbf{r}^{\prime}}^{Q Q}=\int_{0}^{\infty} d t^{\prime} \operatorname{Tr}\left[\bar{\rho}_{t} \hat{\mathbf{Q}} \hat{\mathbf{Q}}_{\mathbf{r}}\left(t^{\prime}\right)\right],
\end{aligned}
$$

where the total mass and heat fluxes are the microscopic phase functions

$$
\begin{aligned}
\hat{\mathbf{J}} & =\int d \mathbf{r} \hat{\mathbf{J}}_{\mathbf{r}}=\sum_{i} \mathbf{v}_{i} \\
\hat{\mathbf{Q}} & =\int d \mathbf{r} \hat{\mathbf{Q}}_{\mathbf{r}}=\sum_{i} \mathbf{v}_{i} e_{i}+\frac{1}{4} \sum_{i j} \mathbf{F}_{i j}\left(\mathbf{v}_{i}+\mathbf{v}_{j}\right) \mathbf{r}_{i j} .
\end{aligned}
$$

The coefficients (108) depend in a functional form on the fields $n(\mathbf{r}), e(\mathbf{r})$ because of the functional dependence of the relevant ensemble with which we compute the correlations in the Green-Kubo expressions (77). The relevant ensemble $\bar{\rho}_{t}$, in situations where the space variation of the gradients is small, can be approximated with an ordinary equilibrium ensemble $\rho^{\mathrm{eq}}$ with global parameters $\lambda, \beta$ matched at the local values of the fields $n(\mathbf{r}), e(\mathbf{r}) .^{32}$ In this way, the above coefficients become functions of the local values of the fields $n(\mathbf{r})$, $e(\mathbf{r})$. Being computed with the equilibrium ensemble, the correlations become translationally invariant, i.e., independent of the explicit value of $\mathbf{r}$. Of course, they depend on the position implicitly through the dependence on the local fields of the matched equilibrium ensemble. The transport coefficients may be now computed with ordinary molecular simulations. 
To this end, it seems appropriate to avoid the Dirac delta function by averaging the correlation functions over a small volume $V$. In this way, Green-Kubo expressions for the transport coefficients result:

$$
\begin{aligned}
D & =\frac{1}{V} \int d \mathbf{r} \int d \mathbf{r}^{\prime} \mathbf{M}_{\mathbf{r r}^{\prime}}^{J J}=\frac{1}{V} \int_{0}^{\infty} d t \operatorname{Tr}\left[\rho^{\mathrm{eq}} \hat{\mathbf{J}} \hat{\mathbf{J}}_{V}(t)\right], \\
C & =\frac{1}{V} \int d \mathbf{r} \int d \mathbf{r}^{\prime} \mathbf{M}_{\mathbf{r r}^{\prime}}^{J Q}=\frac{1}{V} \int_{0}^{\infty} d t \operatorname{Tr}\left[\rho^{\mathrm{eq}} \hat{\mathbf{J}} \hat{\mathbf{Q}}_{V}(t)\right], \\
K & =\frac{1}{V} \int d \mathbf{r} \int d \mathbf{r}^{\prime} \mathbf{M}_{\mathbf{r r}}^{Q Q}=\frac{1}{V} \int_{0}^{\infty} d t \operatorname{Tr}\left[\rho^{\mathrm{eq}} \hat{\mathbf{Q}} \hat{\mathbf{Q}}_{V}(t)\right] .
\end{aligned}
$$

Here, the

$$
\begin{aligned}
\hat{\mathbf{J}}_{V}(t)= & \sum_{i} \delta_{V}(i) \mathbf{v}_{i}, \\
\hat{\mathbf{Q}}_{V}(t)= & \sum_{i} \delta_{V}(i) \mathbf{v}_{i} e_{i} \\
& +\frac{1}{4} \sum_{i j} \mathbf{F}_{i j}\left(\mathbf{v}_{i}+\mathbf{v}_{j}\right) \int_{0}^{1} d \epsilon \mathbf{r}_{i j} \delta_{V}\left(\mathbf{r}_{j}-\epsilon \mathbf{r}_{i j}\right),
\end{aligned}
$$

where $\delta_{V}(\mathbf{r})$ takes the value 1 if particle $\mathbf{r}$ is inside the volume $V$ and zero otherwise. We expect that the value of the transport coefficients is insensitive to the particular value of $V$ provided that $V$ is much smaller than the simulation box (in order to avoid surface effects). The transport coefficients $D, S, K$ are functions of the local thermodynamic state $\lambda(\mathbf{r})$, $\beta(\mathbf{r})$ of the system, through the equilibrium ensemble $\rho^{\text {eq }}$ that has its intensive parameters matched to the local values. The dissipative matrix is positive definite because for any vector $(a, b)$, we have

$$
(a, b)\left(\begin{array}{ll}
D & C \\
C & K
\end{array}\right)\left(\begin{array}{l}
a \\
b
\end{array}\right)=\frac{1}{V} \int_{0}^{\infty} d t \operatorname{Tr}\left[\rho^{\mathrm{eq}} \hat{\mathbf{R}} \hat{\mathbf{R}}(t)\right]>0,
$$

where $\hat{\mathbf{R}}=a \hat{\mathbf{J}}+b \hat{\mathbf{Q}}$. The last inequality follows from the Wiener-Khintchine theorem that ensures that the time integral of an auto-correlation is always positive. The eigenvalues being positive, translate into the conditions $D>0, K>0$, $D K>C^{2}$ for the transport coefficients. The coefficient $D(\mathbf{r})$ is a self-diffusion coefficient, the coefficient $C(\mathbf{r})$ that couples thermal gradients with density variations in time is connected to a Soret coefficient, while $K(\mathbf{r})$ is related to the thermal conduction coefficient. It should be stressed that the above equations are for a single component fluid, not a mixture.

\section{DISCUSSION}

Equations (107) are one of the main results of the present paper. They constitute the generalization of time-dependent DFT to non-isothermal situations for simple fluids. It may look that these equations have the deceptively simple appearance of the equations of thermo-diffusion obtained in standard irreversible thermodynamics. ${ }^{45}$ However, it should be stressed that the present equations may describe structural changes at nanoscales because the entropy functional is in general able to describe the structured density and energy profiles. Note that the local approximation adopted for the transport coefficients does not obliges us to take a local approximation for the entropy functional. Local transport coefficients seem to be a good approximation whenever the derivatives of the entropy functional are smooth. However, the derivatives of the conjugate variables being smooth do not imply that the fields $n(\mathbf{r}), e(\mathbf{r})$ are almost constant. On the contrary, they will display the ordered structure of a molecular fluid at nanoscales. Of course, if one is not interested in these ordering effects, we may use the approximate local functional (101) that wipes off all molecular correlations and will give a poor description of the behaviour of the system at molecular length scales. The local model for the entropy is useful if one is interested in large scale dynamics. By using the local entropy functional, one recovers the usual linear irreversible thermodynamic result based on the local equilibrium assumption..$^{45}$

In the present formulation, that has been derived for an isolated Hamiltonian system, only the tendency towards equilibrium can be described. This implies that the evolution equations describe the tendency of the fields $n(\mathbf{r}), e(\mathbf{r})$ towards the equilibrium state that maximizes the entropy functional subject to the conservation restrictions (79). The entropy functional contains all the information about profiles and correlations, and, therefore, the succession of states towards equilibrium display the non-equilibrium local structure of the system. Examples of systems for which this may be useful are the dynamics of liquid/solid systems with sudden increase or decrease of temperature in some region of the system for which the decay towards the new equilibrium state compatible with the new total energy is described. Of course, most systems of experimental interest are not isolated but rather interacting with the surroundings, either with some global forcing or through boundary conditions. In particular, if stationary temperature gradients are to be established, contact with thermal reservoirs needs to be accounted for in the description. The same framework of projection operators used in the present work can be used in order to include the description of the interaction with the "uninteresting surrounding" that corresponds to a thermal bath. In this case, additional terms accounting for the interaction with the thermal bath appear in the dynamic equations. These terms play the role of "microscopically derived" boundary conditions. We will present this finding in a future publication, but the main message is that the structure of the equations in the bulk is the same as the one that we present in this paper. In this way, possible applications of the present work arise in nanoscale dynamics of melting solids, non-isothermal flow in nano-capillaries (i.e., carbon nanotubes), evaporation of nanodroplets, etc.

The scope of the present theory is limited by the very selection of $n(\mathbf{r}), e(\mathbf{r})$ as relevant variables. In a Markovian description like the one advocated here, this selection implicitly assumes that any other variable in the system is sufficiently fast as compared to the time scale of evolution of $n(\mathbf{r}), e(\mathbf{r})$. Otherwise, the memory kernels would contain long lived contributions and the Markovian assumption leading to memoryless partial differential equations would not apply. In particular, no convective motion should be present in the system, in the sense that any motion is rapidly relaxed towards zero velocity, in a time scale much shorter than the evolution of 
the density and energy densities. If this is not the case, then it is necessary to include an additional conserved variable, the momentum density field. The resulting description leads to the equations of non-isothermal functional hydrodynamics and will be discussed elsewhere.

\section{ACKNOWLEDGMENTS}

P.E. acknowledges the hospitality and support of the Freiburg Institute for Advanced Studies and BIFI. Financial support under MICINN projects FIS2010-22047-C05-01, FIS2010-22047-C05-03, and MODELICO-S2009/ESP-1691 (Comunidad de Madrid) are greatly acknowledged.

${ }^{1}$ J. Gibbs, Elementary Principles in Statistical Mechanics (Yale University Press, 1902/Dover, New York, 1960).

${ }^{2}$ E. Jaynes, Phys. Rev. 106, 620 (1957).

${ }^{3}$ K. Kawasaki and J. Gunton, Phys. Rev. A 8, 2048 (1973).

${ }^{4}$ H. Mori, Prog. Theor. Phys. 33, 423 (1965).

${ }^{5}$ R. Zwanzig, Phys. Rev. 124, 983 (1961).

${ }^{6}$ J. Kirkwood, F. Buff, and M. Green, J. Chem. Phys. 17, 988 (1949).

${ }^{7}$ M. Green, J. Chem. Phys. 20, 1281 (1952).

${ }^{8}$ R. Kubo, J. Phys. Soc. Jpn. 12, 570 (1957).

${ }^{9}$ B. Robertson, Phys. Rev. 144, 151 (1966).

${ }^{10}$ D. Zubarev, Fortschr. Phys. 18, 125 (1970).

${ }^{11}$ K. Kawasaki, J. Stat. Phys. 123, 711 (2006).

${ }^{12}$ H. B. Callen, Thermodynamics (John Wiley \& Sons, New York, 1960).

${ }^{13}$ R. Evans, Adv. Phys. 28, 143 (1979).

${ }^{14}$ P. Hopkins, A. J. Archer, and R. Evans, J. Chem. Phys. 129, 214709 (2008).

${ }^{15}$ H. Löwen, J. Phys.: Condens. Matter 15, V1 (2003).

${ }^{16}$ G. Kahl and H. Löwen, J. Phys.: Condens. Matter 21, 464101 (2009).

${ }^{17}$ J. Lutsko, Adv. Chem. Phys. 144, 1 (2010).
${ }^{18}$ N. Mermin, Phys. Rev. 137, A1441 (1965).

${ }^{19}$ U. Marconi and P. Tarazona, J. Phys.: Condens. Matter 12, A413 (2000).

${ }^{20}$ A. J. Archer and R. Evans, J. Chem. Phys. 121, 4246 (2004).

${ }^{21}$ B. Goddard, A. Nold, N. Savva, G. Pavliotis, and S. Kalliadasis, Phys. Rev. Lett. 109, 1 (2012).

${ }^{22}$ H. T. Davis, J. Chem. Phys. 86, 1474 (1987).

${ }^{23}$ L. Pozhar and K. Gubbins, J. Chem. Phys. 99, 8970 (1993).

${ }^{24}$ Z. Guo, T. S. Zhao, and Y. Shi, Phys. Fluids 18, 067107 (2006).

${ }^{25}$ J. Anero and P. Español, EPL 78, 50005 (2007).

${ }^{26}$ A. J. Archer, J. Chem. Phys. 130, 014509 (2009).

${ }^{27}$ U. Marconi and S. Melchionna, J. Phys.: Condens. Matter. 22, 364110 (2010).

${ }^{28}$ K. H. Hughes and I. Burghardt, J. Chem. Phys. 136, 214109 (2012).

${ }^{29}$ J. Karkheck and G. Stell, Phys. Rev. A 25, 3302 (1982).

${ }^{30}$ M. Trovato and L. Reggiani, Phys. Rev. B 73, 245209 (2006).

${ }^{31}$ P. Español and H. Löwen, J. Chem. Phys. 131, 244101 (2009).

${ }^{32}$ H. Grabert, Projection Operator Technique in Non-Equilibrium Statistical Mechanics (Springer Verlag, 1982).

${ }^{33}$ M. Schmidt, Phys. Rev. E 84, 051203 (2011).

${ }^{34}$ R. Wittkowski, H. Löwen, and H. Brand, J. Chem. Phys. 137, 224904 (2012).

${ }^{35}$ J. Percus, J. Stat. Phys. 52, 1157 (1988).

${ }^{36}$ J. Caillol, J. Phys. A 35, 4189 (2002).

${ }^{37}$ W. Dwandaru and M. Schmidt, Phys. Rev. E 83, 1 (2011).

${ }^{38}$ C. Hijón, E. Vanden-Eijnden, R. Delgado-Buscalioni, and P. Español, Faraday Discuss. 144, 301 (2010).

${ }^{39}$ J. White and A. González, J. Phys.: Condens. Matter 14, 11907 (2002).

${ }^{40}$ J.-P. Hansen and I. McDonald, Theory of Simple Liquids (Academic Press, London, 1986).

${ }^{41}$ J. F. Lutsko, J. Chem. Phys. 134, 164501 (2011).

${ }^{42}$ P. Tarazona, Phys. Rev. A 31, 2672 (1985).

${ }^{43}$ T. Ramakrishnan and M. Yussouff, Phys. Rev. B 19, 2775 (1979).

${ }^{44}$ R. Roth, J. Phys.: Condens. Matter 22, 063102 (2010).

${ }^{45}$ S. R. de Groot and P. Mazur, Non-equilibrium Thermodynamics (North Holland Publishing Company, Amsterdam, 1964). 